\title{
Smart Cities at the Forefront of the Future Internet
}

\author{
José M. Hernández-Muñoz ${ }^{1}$, Jesús Bernat Vercher ${ }^{1}$, Luis Muñoz ${ }^{2}$, José A. Galache², \\ Mirko Presser ${ }^{3}$, Luis A. Hernández Gómez ${ }^{4}$, and Jan Pettersson ${ }^{5}$ \\ ${ }^{1}$ Telefonica I+D, Madrid, Spain \\ \{jmhm, bernat\}atid.es \\ ${ }^{2}$ University of Cantabria, Santander, Spain \\ \{luis, jgalache\}@tlmat.unican.es \\ ${ }^{3}$ Alexandra Institute, Aahrus, Denmark \\ mirko.presser@alexandra.dk \\ ${ }^{4}$ Universidad Politécnica Madrid, Spain \\ luisalfonso.hernandez@upm.es \\ ${ }^{5}$ Centre for Distance-Spanning Technology, Skellefteå, Sweden \\ jan.petterssonaltu.se
}

\begin{abstract}
Smart cities have been recently pointed out by M2M experts as an emerging market with enormous potential, which is expected to drive the digital economy forward in the coming years. However, most of the current city and urban developments are based on vertical ICT solutions leading to an unsustainable sea of systems and market islands. In this work we discuss how the recent vision of the Future Internet (FI), and its particular components, Internet of Things (IoT) and Internet of Services (IoS), can become building blocks to progress towards a unified urban-scale ICT platform transforming a Smart City into an open innovation platform. Moreover, we present some results of generic implementations based on the ITU-T's Ubiquitous Sensor Network (USN) model. The referenced platform model fulfills basic principles of open, federated and trusted platforms (FOTs) at two different levels: the infrastructure level (IoT to support the complexity of heterogeneous sensors deployed in urban spaces), and at the service level (IoS as a suit of open and standardized enablers to facilitate the composition of interoperable smart city services). We also discuss the need of infrastructures at the European level for a realistic large-scale experimentally-driven research, and present main principles of the unique-in-the-world experimental test facility under development within the SmartSantander EU project.
\end{abstract}

Keywords: Smart Cities, Sensor and Actuator Networks, Internet of Things, Internet of Services, Ubiquitous Sensor Networks, Open, Federated and Trusted innovation platforms, Future Internet.

\section{Introduction}

At a holistic level, cities are 'systems of systems', and this could stand as the simplest definition for the term. However, one of the most well-known definitions was provided by the EU project 'European Smart Cities' [1]. Under this work, six dimensions 
of 'smartness' were identified (economy, people, governance, mobility, environment, and living).

As the upsurge of information and communication technologies (ICT) has become the nervous system of all modern economies, making cities smarter is usually achieved through the use of ICT intensive solutions. In fact, ICT is already at the heart of many current models for urban development: revamping their critical infrastructure and enabling new ways of city transport management, traffic control or environmental pollution monitoring. The extensive use of ICT is also empowering the development of essential services for health, security, police and fire departments, governance and delivery of public services.

Nevertheless, the main concern with respect to most of these solutions is that its own commercial approach is leading to an unmanageable and unsustainable sea of systems and market islands. From the point of view of the European Commission, there is a need to reach to a high level agreement at an industrial level to overcome this increasing market fragmentation, which prevents solutions of becoming more efficient, scalable and suitable for supporting new generations of services that are not even envisaged nowadays.

Consequently, the successful development of the Smart Cities paradigm will "require a unified ICT infrastructure to allow a sustainable economic growth" [2], and this unified ICT platform must be suitable to "model, measure, optimize, control, and monitor complex interdependent systems of dense urban life" [3]. Therefore in the design of urban-scale ICT platforms, three main core functionalities can be identified:

- Urban Communications Abstraction. One of the most urgent demands for sustainable urban ICT developments is to solve the inefficient use (i.e. duplications) of existing or new communication infrastructures. Due to the broad set of heterogeneous urban scenarios, there will be also a pronounced heterogeneity of the underlying communication layers. So far, through communications abstraction, urban-scale ICT platforms will allow unified communications regardless the different network standards and will enable data transfer services agnostic to the underlying connection protocol. Furthermore, a major challenge in future urban spaces will be how to manage the increasing number of heterogeneous and geographically dispersed machines, sensors and actuators intensively deployed everywhere in the city.

- Unified Urban Information Models. Also related to the huge amount of heterogeneous information generated at urban scale, a unified ICT platform should be built on top of a unified model so that data and information could be shared among different applications and services at global urban levels. This will relay on the articulation of different enriched semantic descriptions, enabling the development of information processing services involving different urban resources and entities of interest. Specific information management policies should also be addressed to ensure the required level of security and privacy of information.

- Open Urban Services Development. Together with unified communications and information, a key functionality of urban ICT Platforms should be to guarantee interoperability at both the application and service levels. Only through open, easyto-use, and flexible interfaces the different agents involved (public administrations, enterprises, and citizens) will be able to conceive new innovative solutions to interact 
with and manage all aspects of urban life in a cost-effective way. This will provide the necessary innovation-enabling capabilities for attracting public and private investments to create products and services which have not yet been envisioned, a crucial aspect for SmartCities to become future engines of a productive and profitable economy.

Once major challenges of unified urban-scale ICT platforms are identified, it is clear that the future development of Smart Cities will be only achievable in conjunction with a technological leap in the underlying ICT infrastructure. In this work we advocate that this technological leap can be done by considering Smart Cities at the forefront of the recent vision of the Future Internet (FI). Although there is no universally accepted definition of the Future Internet, it can be approached as "a socio-technical system comprising Internet-accessible information and services, coupled to the physical environment and human behavior, and supporting smart applications of societal importance" [4]. Thus the FI can transform a Smart City into an open innovation platform supporting vertical domain of business applications built upon horizontal enabling technologies. The most relevant basic FI pillars [11] for a Smart City environment are the following:

- The Internet of Things (IoT): defined as a global network infrastructure based on standard and interoperable communication protocols where physical and virtual "things" are seamlessly integrated into the information network [5].

- The Internet of Services (IoS): flexible, open and standardized enablers that facilitate the harmonization of various applications into interoperable services as well as the use of semantics for the understanding, combination and processing of data and information from different service provides, sources and formats.

- The Internet of People (IoP): envisaged as people becoming part of ubiquitous intelligent networks having the potential to seamlessly connect, interact and exchange information about themselves and their social context and environment.

At this point, it is important to highlight a bidirectional relationship between the FI and Smart Cities: as if, in the one direction, FI can offer solutions to many challenges that Smart Cities face; on the other direction, Smart Cities can provide an excellent experimental environment for the development, experimentation and testing of common FI service enablers required to achieve 'smartness' in a variety of application domains [6]. To fully develop the Smart City paradigm at a wide geographical scope, a better understanding and insight on issues like: required capacity, scalability, interoperability, and stimulation of faster development of new and innovative applications is required. This knowledge must be taken into account to influence the specification of the FI architecture design. The availability of such infrastructures is expected to stimulate the development of new services and applications by various types of users, and to help gathering a more realistic assessment of users' perspective by means of acceptability tests. To this later extent, close to the IoP vision, the Living Labs network [7] based on the user-driven approach is of main relevance, although in this paper we also advocate the need of large, open and federated experimental facilities able to support experimental research in order to gain real feedback at a city scale [8]. 
The rest of the paper is organized as follows: Section 2 discusses how major components of the Future Internet, namely IoT and IoS, can be essential building blocks in future Smart Cities open innovation platforms. Several technical details related to the development of next generation urban IoT platforms are outlined in Section 3. Section 4 discusses the need for realistic urban-scale open and federated experimental facilities, and presents most relevant current initiatives, with special attention to the SmartSantander EU Project. Finally, conclusions and future challenges are given in Section 5 .

\section{IoT and IoS as ICT Building Blocks for Smart Cities}

In the analysis from Forrester Research [9] on the role that ICT will play in creating the foundation for Smart Cities, a smart city is described as one that "uses information and communications technologies to make the critical infrastructure components and services of a city - administration, education, healthcare, public safety, real estate, transportation and utilities - more aware, interactive and efficient. "According to this approach, frequent in research reports, several key ICT technologies can be identified for their benefits on different city "systems":

- Transportation: sensors can be used to manage the mobility needs with an appropriate Intelligent Transport System (ITS) that takes care of congestion, predicts the arrival of trains, buses or other public transportation options; managing parking space availability, expired meters, reserved lanes, etc.

- ICT can be also used for environmental and energy monitoring: sensors that detect when trash pick-ups are needed, or notify authorities about landfill toxicity; energy consumption and emissions monitoring across sectors to improve accountability in the use of energy and carbon, etc.

- Building management: smart meters and monitoring devices can help monitor and manage water consumption, heating, air-conditioning, lighting and physical security. This can allow the development of smart utilities grids with bidirectional flow in a distributed generation scheme requiring real-time exchange of information.

- Healthcare: telemedicine, electronic records, and health information exchanges in remote assistance and medical surveillance for disabled or elderly people.

- Public Safety and Security: sensor-activated video surveillance systems; locationaware enhanced security systems; estimation and risk prevention systems (e.g. sensitivity to pollution, extreme summer heating).

- Remote working and e-commerce services for businesses, entertainment and communications for individuals. Advanced location based services, social networking and collaborative crowd-sourcing collecting citizens' generated data.

By analyzing these different Smart Cities application scenarios, together with the need of a broadband communication infrastructure that is becoming, or starting to be considered, the $4^{\text {th }}$ utility (after electricity, gas and water), two major ICT building blocks of a Smart City can be identified among the main pillars that the FI provides: 
- Recent advances in Sensors and Actuator Networks (SAN) are stimulating massive sensor networks deployments, particularly for the previously described urban application areas. Therefore IoT, essential to the FI, can be invaluable to provide the necessary technological support to manage in a homogeneous and sustainable way the huge amount of sensor and devices connected to the Smart City infrastructure.

- In a complementary vein, only an open and easy-to-use service enablement suite, that allows the efficient orchestration and reuse of applications, can foster new solutions and services to meet the needs of cities and their inhabitants. In this context, IoS evolution must be undoubtedly correlated with IoT advances. Otherwise, a number of future Smart City services will never have an opportunity to be conceived due to the lack of the required links to the real world.

So far, it may be relevant to consider both the benefits and challenges of implementing IoT and IoS at the city scale.

Starting with the benefits of IoT technologies, they are two-fold: on the one hand they can increase the efficiency, accuracy and effectiveness in operation and management of the city's complex ecosystem and, on the other, they can provide the necessary support for new innovative applications and services (the city as an Open Innovation Platform).

In that sense, the FI PPP promoted by the EC [10][11] seeks for the cooperation among the main European stakeholders in order to develop cross-domain Next Generation (NG) IoT platforms suitable to different usage areas and open business models to improve market dynamics by involving third parties in the value chain (SMEs).

Some of the essential functionalities identified as required for NG IoT platforms comprise the support for horizontality, verticality, heterogeneity, mobility, scalability, as well as security, privacy, and trust [12][13]. Cross-domain NG IoT platforms may foster the creation of new services taking advantage of the increasing levels of efficiency attained by the re-use of deployed infrastructures.

Considering now the IoS, it must be stressed that it is widely recognized (see for example [12]) that the real impact of future IoT developments is heavily tied to the parallel evolution of the IoS. So, a Smart City could only become a true open innovation platform through the proper harmonization of IoS and IoT. There can be a long list of potential benefits for Smart Cities' services relaying on the same basic sensed information and a suite of application enablers (i.e. from sensor data processing applications, to enablers for accessing multimedia mobile communications or social networks, etc.). Thus the integration of innovative principles and philosophy of IoS will engage collective end-user intelligence from Web 2.0 and Telco 2.0 models that will drive the next wave of value creation at urban scales, a key aspect typically missing in other technologically-driven initiatives.

The technological challenge of developing the IoS has been assumed at EU level, and actions are being initiated to overcome the undesirable dissociation between technological and service infrastructures [13]. Of particular relevance for Smart Cities scenarios can be the relatively new evolving concept of a Global Service Delivery Platform GSDP under the FOT (Federated, Open and Trusted) platform model [14]. As Figure 1 illustrates, the GSDP vision can represent one single point of access to a federation/network of interoperable urban platforms (including both experimental and deployed IoT platforms). In that way, an increasing number of Smart Cities' services could be searched, 
discovered and composed (following Web 2.0/Telco2.0 principles and including QoS, trust, security, and privacy) in a standard, easy and flexible way. Now that a number of different approaches towards future GSDP are being addressed in several EU research projects such as SOA4ALL, SLA@SOI, MASTER, NEXOF-RA, etc. (as stated in the 2008 FIA meeting: "One project on its own cannot develop a GSDP" [20] [15]), the Smart Cities can represent an extraordinary rich ecosystem to promote the generation of massive deployments of city-scale applications and services for a large number of activity sectors. Furthermore this will enable future urban models of convergent IT/Telecom/Content services, Machine-to-Machine (M2M) services, or entirely new service delivery models simultaneously involving virtual and real worlds.

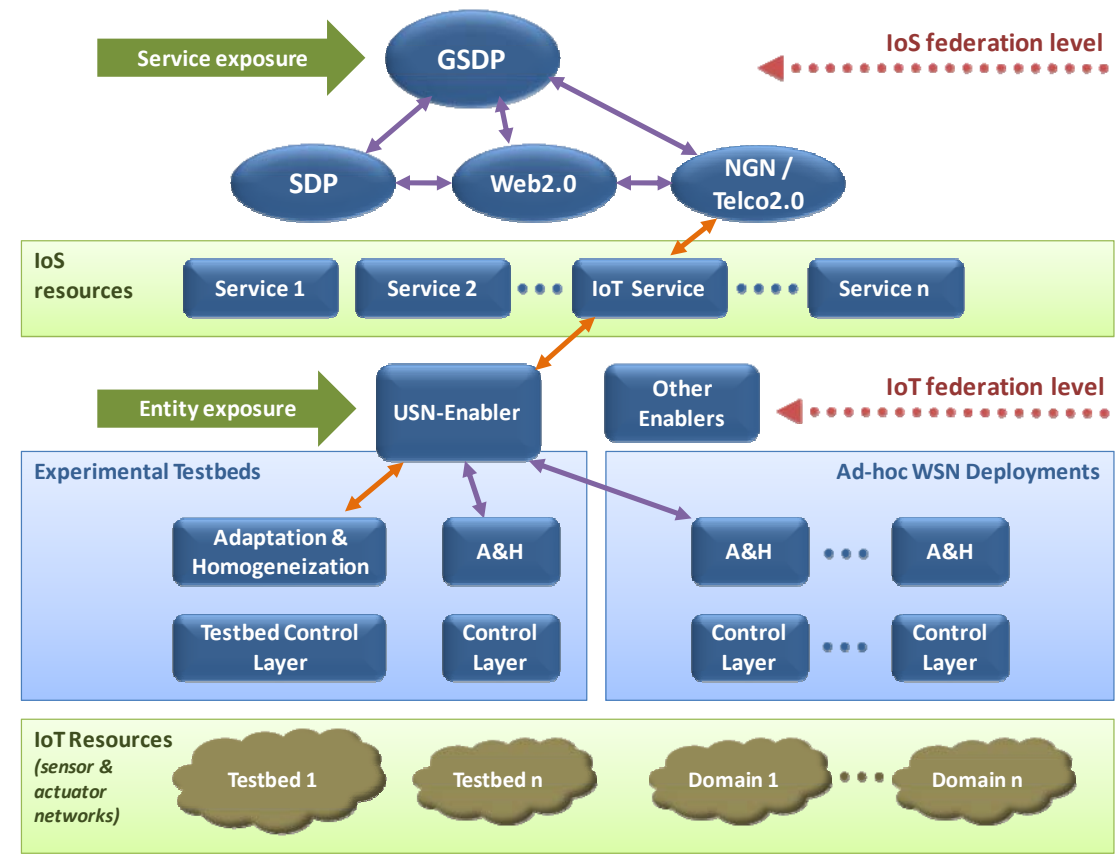

Fig. 1. Global Service Delivery Platform (GSDP) integrating IoT / IoS building blocks

\section{Developing Urban IoT Platforms}

At present, some works have been reported of practical implementations in order to develop IoT platforms inspired by the Ubiquitous Sensor Networks concept from the ITU-T USN Standardization Group [21]. Some research teams have already initiated activities in this line, but there are currently very few references to them in the literature. ITU's USN concept envisions a "technological framework for ambient sensor networks not as simple sets of interconnected networks but as intelligent information infrastructures". The concept translates directly into cities, as they can be considered as one multi-dimensional eco-system, where data is binding the different dimensions, as most aspects are closely related (e.g. environment and traffic, both of them to health, etc.). 


\subsection{USN Functionalities}

The main goal of a USN platform is to provide an infrastructure that allows the integration of heterogeneous and geographically disperse sensor networks into a common technological ground where services can be developed in a cost efficient manner. Consequently, at urban-scale, a USN platform can represent an invaluable infrastructure to have access and manage the huge amount of sensor and devices connected in Smart City environments. Through a set of basic functionalities it will support different types of Smart City services in multiple application areas:

- Sensor Discovery: this functionality will provide services and applications information about all the registered sensors in the city. In that way, a particular service interested in finding information (such as available parking places in a given area) will have access to efficient look-up mechanisms based on the information they provide.

- Observation Storage: many Smart City services will rely on continuously generated sensor data (for example for energy monitoring, video surveillance or traffic control). This functionality will provide a repository where observations / sensors' data are stored to allow later retrieval or processing, to extract information from data by applying semantic annotation and data linkage techniques.

- Publish-Subscribe-Notify: in other cases, services rely on some specific events happening in the city (such as traffic jams or extreme pollution situations). The platform will allow services to subscribe not just to the observations provided by the sensors, but also to complex conditions involving also other sensors and previous observations and measurements.

- Homogeneous Remote Execution capabilities: This functionality allows executing tasks in the sensor/actuator nodes, so city services could either change sensor configuration parameters (i.e. the sensibility of a critical sensor) or to call actuator commands (as, for example, closing a water pipe).

Another important set of capabilities provided by a USN platform is related to the need for a homogeneous representation and access to heterogeneous urban information, as it was discussed in Section 1. In this sense, the main basic principles covered by USN platforms are:

- Unified information modeling: The information should be provided to the Smart City services using a unified information model, regardless of the particular information model used by the sensor technologies deployed through the city infrastructure. This principle should be applied both to the sensor descriptions and to observations.

- Unified communication protocol: given the extension of an urban area, several standards can co-exist to communicate sensors and sensor networks (ZigBee, 6LowPan, ISA-100.11.a, xDSL, GPRS, etc.). Services should be agnostic to the communication protocol used. The platform should provide access to the information regardless the particular underlying communication protocol used. 
- Horizontally layered approach: The platform should also be built following a layered approach, so services and networks are decoupled in order to evolve independently [22]. This capability will allow a seamless link between IoT and IoS, as discussed in Section 2.

Also relevant will be the definition of open APIs, so that USN platforms could provide support for third-party's agents interested in the deployment of different Smart City services, thus allowing federation with different service creation environments and different business processes.

\subsection{USN Architecture for Urban IoT Platforms}

While the new wave of Next Generation IoT platforms are expected to be defined by initiatives and projects like IoT-A [23], the IERC cluster [24] or the emerging PPP IoT Core Platform Working Group discussion [25], multiple different approaches for First Generation IoT-platforms are currently being implemented. In essence, many of them are realizations of the described ITU-T's model. For reference on the current state of the technology, this Section describes a practical USN platform implementation (more details can be found in [22]), integrated into the Next Generation Networks Infrastructures [35], as one of the most remarkable currently reported solutions for advanced IoT platforms. As shown in Figure 2, a functional specialization of the building blocks has been applied in this work.

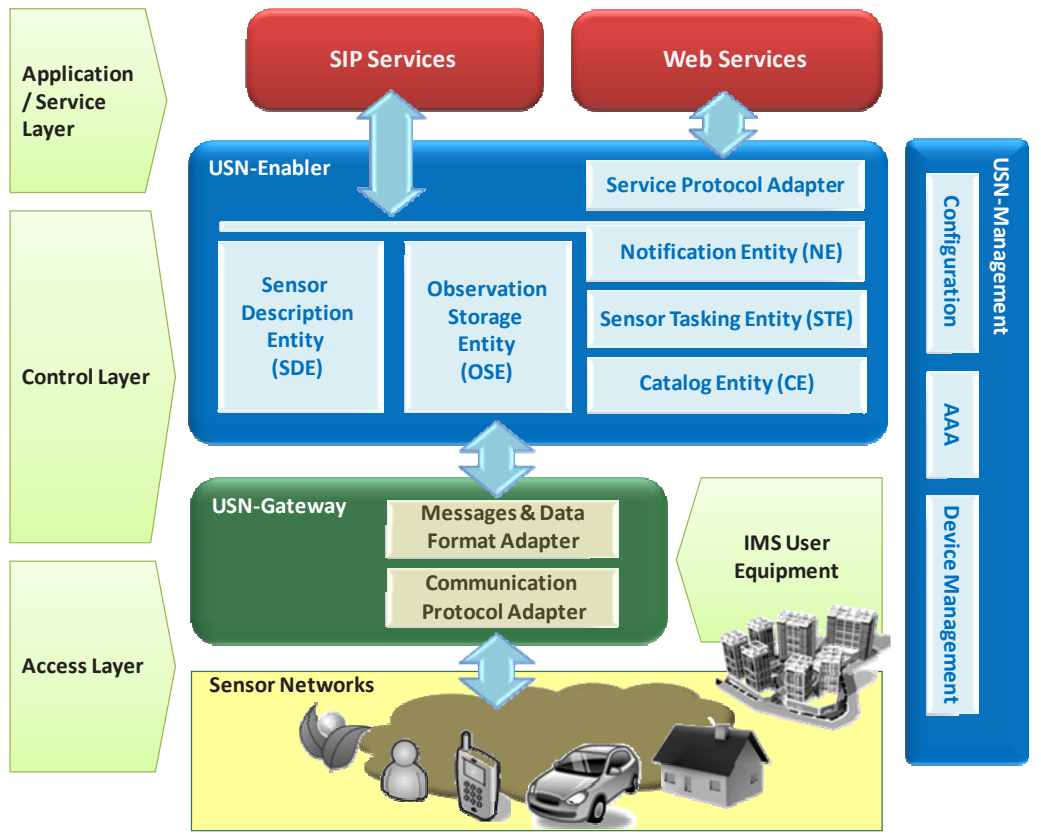

Fig. 2. High-Level Architecture of a USN IoT Platform 
As sketched in the figure, the USN platform is based on two components, the USNEnabler (that interfaces services) and the USN-Gateways (that interacts with Sensor networks). This approach is inspired by the Open Geospatial Consortium (OGC) Sensor Web Enablement (SWE) activity [26]. Its goal is the creation of the foundational components to enable the Sensor Web concept, where services will be capable to access any type of sensors through the web. This has been reflected by a set of standards used in the platform (SensorML, Observation \& Measurements, Sensor Observation Service, Sensor Planning Service, Sensor Alert Service and Web Notification Service [26]). Besides the SWE influence, the USN-Enabler relays on existing specifications from the OMA Service Environment (OSE) [27] enablers (such as presence, call conferencing, transcoding, billing, etc.). Especially important has been the Presence SIMPLE Specification (for publish and subscribe mechanisms to sensor information) and XML Document Management, also known as XDM (for XML information modeling in Service Enablers).

The USN-Gateway represents a logical entity acting as data producers to the USNEnabler that implements two main adaptation procedures to integrate physical or logical Sensor and Actuator Networks (SANs):

- Communication Protocol Adaptation. As a connection point between two networks (sensors networks deployed throughout the city and the core IP communication network), the main responsibility is to provide independence from the communication protocol used by the sensor networks.

- Sensor Data Format Adaptation. This functionality is intended to provide USNEnabler both SensorML (meta-information) and O\&M (observation \& measurements) data from specific SANs data (i.e. ZigBee).

Adaptation and Homogenization are two key requirements for the USN Platform aiming at its integration with different Smart Cities' testbeds and experimental deployments. They are also essential requirements for a successful seamless integration, and the proper basement for the new heterogeneous sensor network infrastructures needed to enable an evolving FI based on the IoT and IoS paradigms.

Functionalities required to support services are offered both in synchronous and asynchronous mode by the USN-Enabler through the following entities:

- The Sensor Description Entity (SDE) is responsible for keeping all the information of the different city sensors registered in the USN-Platform. It uses the SensorML language.

- The Observation Storage Entity (OSE) is responsible for storing all the information that the different sensors have provided to the platform related to the different Smart City resources and Entities of Interest. This information is stored using the OGC® O\&M language.

- The Notification Entity (NE) is the interface with any sensor data consumer that require filtering or information processing over urban-generated data. The main functionalities provided by this entity are the subscription (receive the filter that will be applied), the analysis of the filters (analyze the filter condition) and the notification (notify the application when the condition of the filter occurs). 
- The Sensor Tasking Entity (STE) allows services to perform requests operations to the sensor network, like for example a request to gather data, without the need to wait for an answer. The service will receive an immediate response and, when the desired data gets available it will receive the corresponding alert. This is mainly used for configuration and for calling actuators.

- The Service Protocol Adapter (SPA) provides protocol adaptation between the Web Services and SIP requests and responses.

- The Catalogue and Location Entity (CLE) provides mechanisms in a distributed environment to discover which of the different instances of the entities is the one performing the request a user might be interested in. For example, in an architecture where several Sensor Description Entities (SDEs) exist, a client might be interested in a particular sensor deployed in a particular city zone. The client should interrogate the CLE to know which particular existing SDEs in the requested urban area contain the information needed.

\section{The Need of Urban Scale Experimental Facilities}

Experimentally-driven research is becoming more and more important in ICT research. When designing heterogeneous large scale systems, difficulties arise in modeling the diversity, complexity and environmental conditions to create a realistic simulation environment. The consequence is clear: simulation results can only give very limited information about the feasibility of an algorithm or a protocol in the field.

In many cases, due to practical and outside plant constraints, a number of issues arise at the implementation phase, compromising the viability of new services and applications. Most of these problems are related to scalability aspects and performance degradation. The level of maturity achieved at the networking level, despite the fact that they can be further improved, foresees an increasing necessity of additional research activity at the sensor and context information management level [17]. Nevertheless, FI research is no longer ending at the simulation stage. Advances in sensor networking technologies need field validation at large scales, also posing new requirements on the experimentation facilities. Besides, new cross-layer mechanisms should be introduced to abstract the networking level from the higher ones, so new services and information management activities can be performed over heterogeneous networking technologies. This increasing demand to move from network experimentation towards service provisioning requirements does not just apply to the Smart Cities field, but also in a more generic way it is common to most FI experimentation areas.

\subsection{Smart Cities as Open Innovation Platforms}

To perform reliable large scale experimentation, the need of a city scale testbed emerges. Setting such an experimental facility into a city context has several reasons: the first one is the extent to which the necessary infrastructure of a Smart City will rely on technologies of the IoT. The resulting scale and heterogeneity of the environment makes it an ideal environment for enabling the above mentioned broad range of experi- 
mentation needs. Furthermore, a city can serve as an excellent catalyst for IoT research, as it forms a very dense techno-social eco-system. Cities can act as invaluable source of challenging functional and non-functional requirements from a variety of problem and application domains (such as vertical solutions for the environment control and safety, horizontal application to test network layers, content delivery networks, etc.). They provide the necessary critical mass of experimental businesses and end-users that are required for testing of IoT as well as other Future Internet technologies for market adoption. This new smart city model can serve as an excellent incubator for the development of a diverse set of highly innovative services and applications [18].

For all these reasons, systems' research in ICT needs more powerful and realistic tools, which can only be provided by large-scale experimental facilities. At a urban scale, this approach yields to a rising importance of non-technical practical issues, like interworking at the organizational level, or even administrative and political constraints. There are very few initiatives addressing the creation of such smart city environments. Some examples are Oulu in Finland [28], Cambridge, Massachusetts [29], or Friedrichshafen, Germany [30]. Most recent and interesting initiatives are Sense Smart City in Skellefteå, Sweden [31], and SmartSantander [32] at a European level. The first one is a Swedish project designed to conduct research, create new business opportunities and sustainably increase ICT research and innovation capability with specific objective to make urban cities/areas "smarter". The project will generate new and better ICT solutions that instrument urban areas to gather and combine information (energy, traffic, weather, events, activities, needs and opinions) continuously as well as "on-demand". This will enable city environments to become "smarter", as more adaptive and supportive environment, for people as well as organizations.

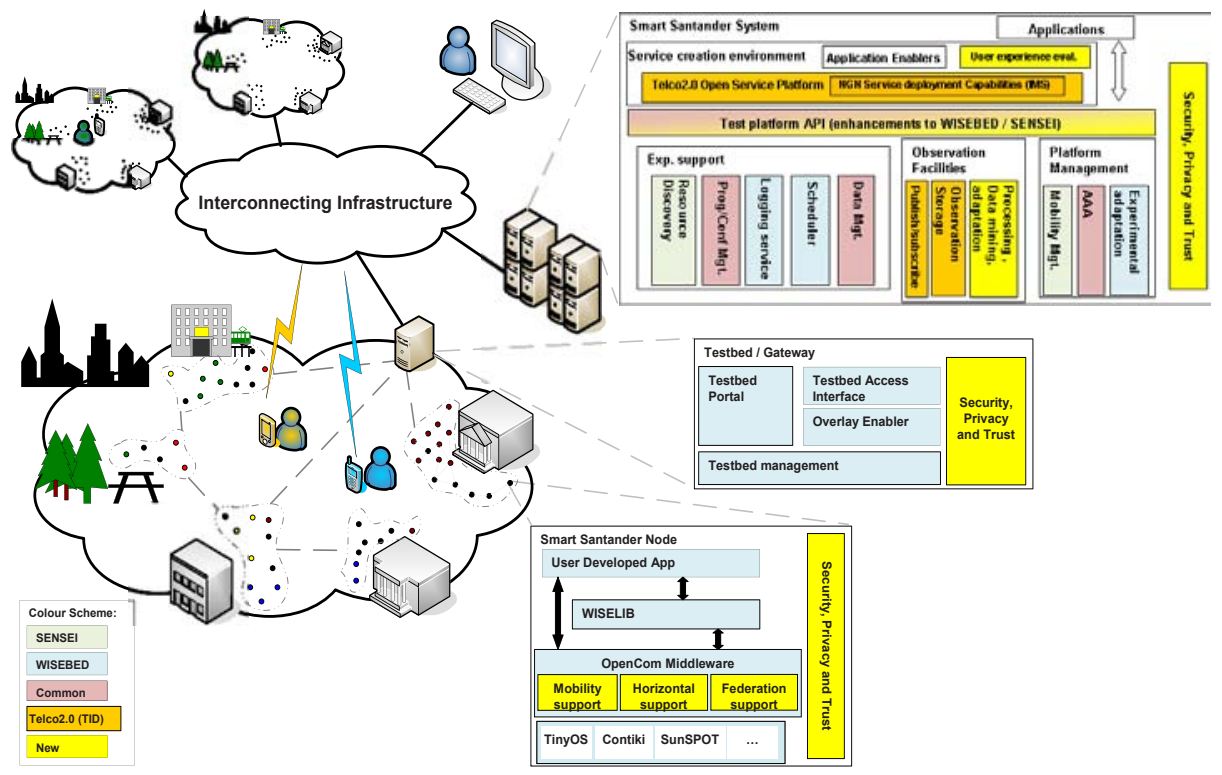

Fig. 3. SmartSantander: A city-scale platform architecture 


\subsection{SmartSantander Experimental Research Facility}

At urban scale, SmartSantander [32] represents the most challenging reference nowadays, and aims at creating a unique-in-the-world European experimental test facility for the research and experimentation of architectures, key enabling technologies, services and applications for the IoT. The facility will allow large-scale experimentation and testing in a real-world environment. The infrastructure will be mainly deployed in Santander in the North of Spain, with nodes in Guildford, UK; Lübeck, Germany; Belgrade, Serbia; Aahrus, Denmark and Melbourne, Australia. Apart from providing state of the art functionalities, the facility will count with advanced capabilities to offer support for experimentation of different FI components. Some of these additional functionalities that are required to support experimental activities can be identified in Figure 3. Primarily, the platform is being designed to allow the experimental evaluation of new research results and solutions in realistic settings. By conception, it will also make possible the involvement of real end-users in the first service design phases, applying user-driven innovation methodologies. Furthermore, it will be also used to provide real services to citizens. SmartSantander experimental facility is not envisaged as a closed, standalone system. Instead, it is being designed to become an open experimental research facility that can be easily expanded and federated with other similar installations (i.e. through OneLab2 [34]).

A key aspect in SmartSantander project is the inclusion of a wide set of applications. Application areas are being selected based on their high potential impact on the citizens as well as to exhibit the diversity, dynamics and scale that are essential in advanced protocol solutions, and will be able to be evaluated through the platform. Thus, the platform will be attractive for all involved stakeholders: industries, communities of users, other entities that are willing to use the experimental facility for deploying and assessing new services and applications, and Internet researchers to validate their cutting-edge technologies (protocols, algorithms, radio interfaces, etc.).

Several use cases are currently under detailed analysis for their experimental deployment taking into account relevant criteria from local and regional authorities. An illustrative list of these use cases is:

- Monitoring several traffic-related events in the city such as: dynamic occupation, mapping and control of limited parking zones, places for disabled people, load/unload areas restricted to industrial; as well as traffic management services: creation of corridors for emergency vehicles, ecoways enablement proposing alternative routes for vehicles based on pollution monitoring in different city zones.

- Tracking and monitoring of people with disabilities, especially mental disorders (Down syndrome, Alzheimer's disease, dementia, etc.) or heart disease that may require constant monitoring by their families or physicians.

- Alert services that, orchestrating several services such as such eHealth, environmental monitoring, traffic control and communication services, will inform and/or alert citizens of different critical situations (i.e. urgent medical attention, city services recommendations, etc.)

- Tourism information in different parts of the city through mobile devices using visual and interactive experiences and in different languages. 
- Video monitoring for traffic areas, beach areas and specific events in public places, such as airports, hotels, train stations, concerts and sport stadiums.

- Smart metering monitoring in buildings and houses for electric energy, water and gas control consumption, including real-time information to the citizens on their own consumption and environmental impact.

Based on these, and future, use cases a main goal in SmartSantander project will be to identify a detailed set of functional (required capabilities) and non-functional (required constraints) requirements for a urban-scale ICT platform. A major challenge will be to leverage on state-of-the-art experimental, research and service oriented initiatives on both IoT and IoS areas as WISEBED [25], SENSEI [8] and the USN IoT Platform (presented in Section 3) including Web 2.0 and Telco 2.0 design principles. Additionally, the requirements elicitation process in SmartSantander will also consider the following viewpoints: the FIRE testbed user, the service provider, the service consumers (citizens), the SmartSantander facility administrators, and individual testbed administrators. The SmartSantander initial architectural model specifies the subsystems (collectively, the SmartSantander middleware) that provide the functionality described by these requirements and is expected to accommodate additional requirements coming up from the different smart city services (use cases). A set of basic subsystems can be identified: i) Access Control and IOT Node Security subsystem, ii) Experiment Support Subsystem, iii) the Facility Management Support Subsystem, and iv) the Application Support Sub-system. The architectural reference model also specifies, for each sub-system, required component deployments on the IoT nodes, and component interactions and information models used to fulfill the subsystem's functionality.

In summary, it can be said that main benefits underlying the SmartSantander partnership is to fuel the use of its urban-scale experimentation facility among the scientific community, end users and service providers. This will not only reduce the technical and societal barriers that prevent the IoT concept to become an everyday reality, but also will attract the widest interest and demonstrate the usefulness of the SmartSantander platform.

Finally, it must be noticed that financial aspects are of the utmost importance considering the investment required to deploy city scale testbeds. For this reason, apart from serving to its research purposes, it is essential, when planning a Smart City open innovation platform, to introduce requirements allowing the support of real life services simultaneously. This will be very useful to open new business opportunities and, at least and not less important, provide the means to guarantee its day by day maintenance.

\section{Conclusions}

Future Internet potential, through IoT and IoS, for creating new real-life applications and services is huge in the smart city context. First time success of large IoT deployments is seriously jeopardized by the lack of testbeds of the required scale, and suitable for the validation of recent research results. Many existing testbeds just offer experi- 
mentation and testing limited to small domain-specific environments or application specific deployments. While those may suffice as proof-of-concepts, they do not allow conclusive experimentation with the developed technologies and architectural models, evaluation of their performance at an adequate scale under realistic operational conditions and, validation of their viability as candidate solutions for real life IoT scenarios.

At present, some practical implementations of advanced USN platforms [22] have been successfully demonstrated in real deployments for smart metering services, smart places scenarios, and environmental monitoring systems. Ongoing activities are extending its scope to broader M2M scenarios, and large scale deployments for experimental smart urban spaces. The described implementation has shown a big potential to create a fan of new services, providing the key components required to intertwining IoT and IoS worlds. Referred IoT USN platform is currently being evolved with the addition of new capabilities, and integrated within other components being previously developed by the EU projects SENSEI [8] and WISEBED [33] to implement a city scale infrastructure for IoT technologies experimentation within the SmartSantander project. In this project, a large infrastructure of about 20,000 IoT devices is addressed. Currently, the deployment of the first 2,000 sensors in the urban environment is been carried. Non-technical aspects are also of a big importance. The cardinality of the different stakeholders involved in the smart city business is so big that many non-technical constraints must be considered (users, public administrations, vendors, etc.). In this sense, what may be evident from a purely technique perspective it is not so clear when politics and business meet technology. Besides, and although market claims its readiness for supporting a vast range of sensing capabilities as well as the corresponding end-user services, the real situation is quite far different. Nowadays, there are no field experiences across the world allowing assessing, in the short term, the behavior of massive wireless sensor deployments.

Acknowledgements. Although only a few names appear on this paper, this work would not have been possible without the contribution and encouragement of many people, particularly all the enthusiastic team of the SmartSantander project, partially funded by the EC under contract number FP7-ICT-257992.

Open Access. This article is distributed under the terms of the Creative Commons Attribution Noncommercial License which permits any noncommercial use, distribution, and reproduction in any medium, provided the original author(s) and source are credited.

\section{References}

1. Smart Cities, Ranking of European medium-sized cities, http: / /www. smart-cities.eu/

2. The ICT behind cities of the future, http://Www.nokiasiemensnetworks.com/ news-events / publications/unite-magazine-february-2010/the-ictbehind-cities-of-the-future

3. Simonov, M.: Future Internet applications relevant for smart cities, an ICT application area example: smart \& proactive energy management, Open Innovation by FI-enabled services, Brussels, 15 January (2010) 
4. Position Paper: Research Challenges for the Core Platform for the Future Internet. In: M. Boniface, M. Surridge, C.U (Eds.) http://ec.europa.eu/information_society/ activities/foi/library/docs/fippp-research-challenges-for-coreplatform-issue-1-1.pdf

5. Sundmaeker, H., Guillemin, P., Friess, P., Woelfflé, S. (eds.): Vision and Challenges for Realising the Internet of Things, CERP-IoT, March 2010. European Commission, Brussels (2010)

6. Future Internet Assembly 2009, Stockholm, Sweden (November 2009), http://ec. europa.eu/information_society/activities/foi/library/docs/fi-stockholm-report-v2.pdf

7. The European Network of Living Labs, http: / /www. openlivinglabs.eu/

8. SENSEI - Integrating the Physical with the Digital World of the Network of the Future. State of the Art - Sensor Frameworks and Future Internet (D3.1). Technical report (2008)

9. Belissent, J.: Getting Clever About Smart Cities: New Opportunities Require New Business Models, 2 November 2010. Forrester Research (2010)

10. EC FI-PPP: http://ec.europa.eu/information_society/activities/foi/ lead/fippp/index_en.htm

11. Towards a Future Internet Public Private Partnership, Usage Areas Workshop, Brussels, 3 March (2010), http: / /ec.europa.eu/information_society/activities / foi / events / fippp3 / fi-ppp-workshop-report-final .pdf

12. Real World Internet (RWI) Session, FIA meeting, Prague (May 2009), http://rwi.future-internet.eu/index.php/RWISession_Prague

13. COM: A public-private partnership on the Future Internet. Brussels, 28 October (2009), http://ec.europa.eu/information_society/activities/foi/library/docs/ fi-communication_en.pdf

14. DG INFSO Task Force on the Future Internet Content.Draft Report of the Task Force on InterdisciplinaryResearch Activities applicable to the Future Internet, Version 4.1 of 13.07.2009 (2009), http: / forum. future-internet.eu

15. NESSI Strategic Research Agenda, http://www.nessi-europe.com/files/ResearchPapers/NESSI_SRA_VOL_3.pdf

16. Gluhak, A., Bauer, M., Montagut, F., Stirbu, V., Johansson, M., Bernat-Vercher, J., Presser, M.: Towards an architecture for a Real World Internet. In: Tselentis, G., et al. (eds.) Towards the Future Internet, IOS Press, Amsterdam (2009)

17. Fisher, S.: Towards an Open Federation Alliance. The WISEBED Consortium. Lulea, July 2nd, 2009.22. In: Balazinska, M., et al. (eds.) Data Management in the Worldwide Sensor Web. IEEE PERVASIVE computing, April-June (2007)

18. Panlab Project, Pan European Laboratory Infrastructure Implementation, http: / / www. panlab. net/fire.html

19. Global service delivery platform (GSDP) for the future internet: What is it and how to use it for innovation?, http://services. future-internet.eu/images/d/d4/Report GSDPpane1-FISO-FIA-Madrid-draftะ2Breqs.pdf

20. Future Internet Assembly, Meeting Report, Madrid, Spain, 9th-10th December (2008), http://ec.europa.eu/information_society/activities/foi/library/ docs/madrid-conference-report-v1-1.pdf

21. ITU TSTAG: A preliminary study on the Ubiquitous Sensor Networks. TSAG-C 22-E (February 2007)

22. Bernat, J., Marín, S.: González. A., Sorribas, R., Villarrubia, L., Campoy, L., Hernández, L. Ubiquitous Sensor Networks in IMS: an Ambient Intelligence Telco Platform. ICT Mobile Summit, 10-12 June, Stockholm (2008)

23. Internet of Things Architecture project, http: / /www. iot-a.eu/public/front-page 
24. IoT European Research Cluster, http: / /www. internet-of-things-research.eu/

25. White paper on the FI PPP definition (Jan. 2010), http://wWw. future-internet. eu/fileadmin/initiative_documents/Publications/White_Paper/EFII_ White_Paper_2010_Public.pdf

26. Botts, M., Percivall, G., Reed, C., Davidson, J.: "OGC Sensor Web Enablement: Overview and High Level Architecture", Open Geospatial Consortium Inc. White Paper Version 3 (2007)

27. OMA Service Environment Archive, http://www.openmobilealliance.org/ technical/release_program/ose_archive.aspx

28. Oulu Smart City, http: / /www. ubiprogram.fi/

29. Cambridge (MA) Smart City, http: / /www. citysense.net/

30. Friedrichshafen Smart City, http: / /www. telekom.com/dtag/cms/content/dt/en/395380

31. Sense Smart City project, http://sensesmartcity.org/

32. SmartSantander project, FP7-ICT-2010-257992, http: / /www. smartsantander eu

33. WISEBED - Wireless Sensor Network Testbeds, http://www. wisebed.eu

34. OneLab2, ONELAB project, http: / /www. onelab.eu/

35. NGNI, http://www. fokus. fraunhofer.de/en/ngni/index.html 